\title{
Directed forgetting in pigeons resulting from the reallocation of memory-maintaining processes on forget-cue trials
}

\author{
DAREN H. KAISER \\ University of Kentucky, Lexington, Kentucky \\ LOU M. SHERBURNE \\ Wabash College, Crawfordsville, Indiana \\ and \\ THOMAS R. ZENTALL \\ University of Kentucky, Lexington, Kentucky
}

\begin{abstract}
When procedural artifacts are controlled, it has been difficult to demonstrate directed forgetting in pigeons. However, previous research with pigeons has not allowed for the reallocation of working memory (from forget items to remember items) on forget-cued trials as is possible in human directed forgetting experiments. In the present experiment, directed forgetting was found while controlling for procedural artifacts and allowing the pigeons to reallocate memory resources on forget trials. The results indicate that under these conditions, pigeons have active control over memory processing.
\end{abstract}

Directed forgetting, a phenomenon that is well documented in the human literature (Bjork, 1972; Epstein, 1972; Johnson, 1994), has been used traditionally to show that humans can have active control over memory maintaining processes. Although several procedures have been used to demonstrate directed forgetting in humans, the one of most interest here is the "distinctive forget instruction" procedure (see Johnson, 1994). With this procedure, participants are given a list of words, some followed by a cue that instructs them that memory for that word will later be tested (remember cued), and some followed by a cue that instructs them that memory for that word will not be tested (forget cued). Humans typically show better memory for words that are cued to be remembered than for words that are cued to be forgotten. This finding suggests that humans can exert active control over information processing and memory.

In general, most animals have not been judged capable of similar control over processing and memory. Most animals have traditionally been viewed as passive associators or storers of environmental events (Hull, 1943; Roberts \& Grant, 1976). Recently, however, more cognitive accounts of animal memory have emerged, and it has been suggested that animals are capable of restructuring the input from environmental events and of forming cognitive representations (Roitblat, 1982).

This research was supported by National Science Foundation Grant BNS-9019080 and National Institute of Mental Health Grant MH45979. Correspondence concerning this article should be addressed to T. R. Zentall, Department of Psychology, University of Kentucky, Lexington, KY 40506 (e-mail: zentall@pop.uky.edu).
Although rats and monkeys have been the subject of directed forgetting research (Grant, 1982; Roberts, Mazmanian, \& Kraemer, 1984), most animal research on directed forgetting has been performed with pigeons (Grant, 1981; Kendrick, Rilling, \& Stonebraker, 1981; Maki \& Hegvik, 1980). One often used method of studying directed forgetting in the pigeon is a variation of the delayed matching-to-sample task that has been termed the omission procedure. In one version of this procedure, pigeons are first trained in a three-key operant chamber to match comparison stimuli (e.g., red and green hues) presented on the two side keys to an initial sample stimulus presented on the center key (e.g., a red or green hue). After pigeons acquire the task with no delay, gradually increasing delays are inserted between sample and comparison stimuli, and potentially informative delay cues are presented on the center key - typically for the duration of the delay. Remember delay cues (R-cues) are always followed by comparisons (i.e., a test of sample memory) and sample-matching responses are reinforced. In the omission procedure, forget delay cues (F-cues) are followed immediately by the intertrial interval (ITI), and thus there is no test of sample memory. After the pigeons have achieved adequate performance on the R-cue trial types, they are tested with occasional probe trials. These probe trials involve presentation of the sample stimulus followed by the F-cue, and then a test for sample memory (i.e., presentation of the comparison stimuli). With this procedure, pigeons generally exhibit poor performance on probe trials, while maintaining a high level of matching accuracy on the R-cue trial types (e.g., Kendrick et al., 1981). 


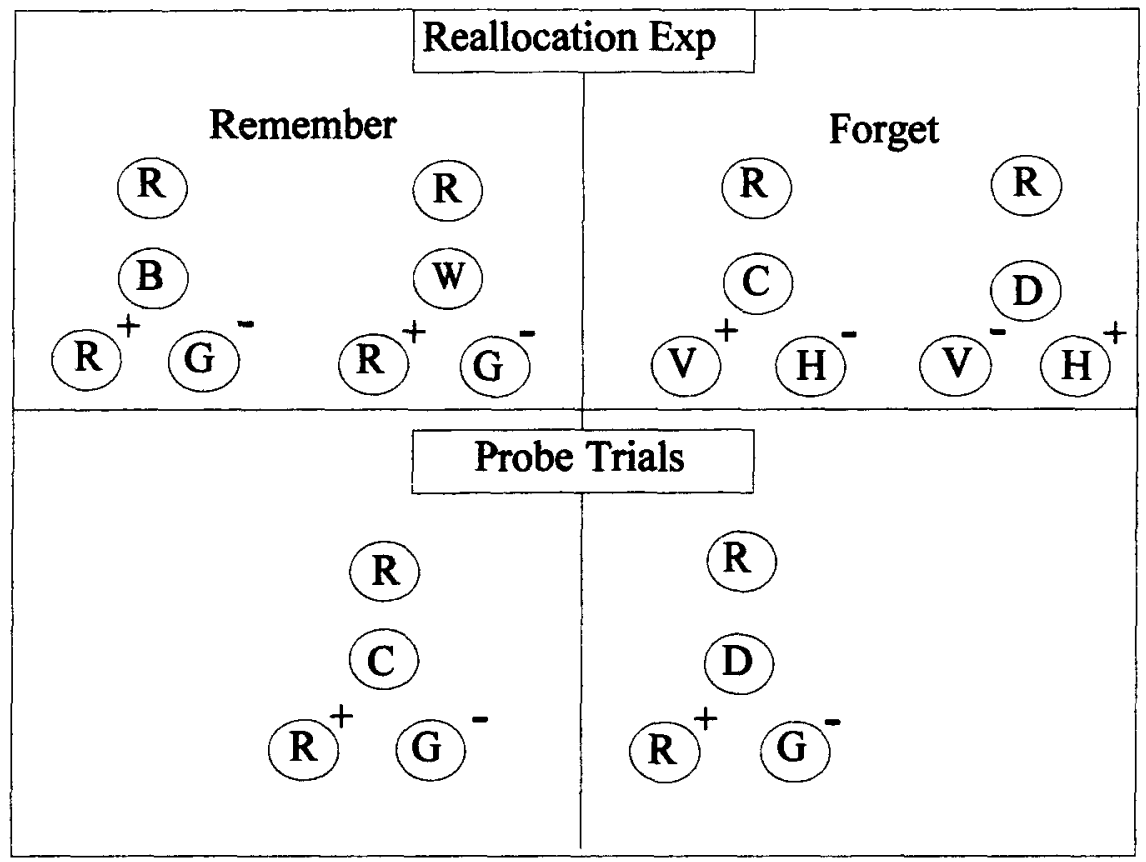

Figure 1. Representation of the Roper, Kaiser, and Zentall (1995) reallocation of memory experiment. Note that only half of the trial types are presented. The other half are similar but involve green samples, and the correct comparison is green where appropriate. $R=\operatorname{red}, \mathbf{G}=$ green, $\mathrm{B}=$ blue, $\mathrm{W}=$ white, $\mathrm{C}=$ circle, $\mathrm{D}=$ dot, $\mathrm{V}=$ vertical, $\mathrm{H}=$ horizontal, $(+)=$ reinforcement, and $(-)=$ nonreinforcement.

This finding of decreased performance on F-cued probe trials as opposed to R-cued trials has been interpreted as evidence that pigeons have active control over memory processing, perhaps by using the $\mathrm{R}$ - and $\mathrm{F}$-cues as signals to rehearse or not to rehearse the sample (Grant, 1981; Santi \& Savich, 1985; Stonebraker \& Rilling, 1981). However, Roper and Zentall (1993) noted that this procedure may produce decrements in matching accuracy on probe trials because of nonmemorial artifacts. First, when the omission procedure is used, there is no response requirement following presentation of the F-cue. Thus, the pigeons may be unprepared to respond on probe trials when comparisons appear following the Fcue (incompatible response pattern). Second, because there is no opportunity for reinforcement following the F-cue, the F-cue could act as a conditioned inhibitor. Conditioned emotional behavior (e.g., Terrace, 1972) produced by the F-cue could interfere with performance on F-cued probe trials. Finally, it is possible that a decrement in performance could occur on F-cue probe trials because the presentation of comparisons following an Fcue is surprising or novel to the pigeon.

The so-called substitution procedure, another method that has been used to study directed forgetting in pigeons, controls for most of these nonmemorial artifacts. The substitution procedure is similar to the omission procedure, but in the substitution procedure the F-cue is followed by the opportunity to obtain reward. For example, a simple simultaneous discrimination is presented following the F-cue (e.g., vertical and horizontal lines are presented on the side keys and the choice of vertical is always reinforced, independently of the sample; Maki \& Hegvik, 1980). With this procedure, pigeons have the opportunity to obtain reinforcement following presentation of the F-cue, and they are also prepared to choose between the two side keys. However, this procedure also typically results in a high level of matching accuracy on F-cued probe trials (i.e., no directed forgetting effect; Kendrick et al., 1981; Maki \& Hegvik, 1980; Maki, Olson, \& Rego, 1981). Thus, there is little evidence for directed forgetting when one controls for these nonmemorial factors. Interestingly, the results of experiments in which this substitution procedure has been used also suggest that surprising comparisons do not disrupt matching accuracy, at least not with the matching-tosample paradigm, because presentation of the unexpected memory test on F-cue probe trials does not result in a decrement in matching accuracy.

These findings suggest that directed forgetting in pigeons may not be due to active memory control by the pigeon, but rather to procedural artifacts (see Roper \& Zentall, 1993; Zentall, Roper, \& Sherburne, 1995). When these procedural artifacts are eliminated, pigeons typically perform as well on F-cued probe trials as they do on $\mathrm{R}$-cued trials.

On the other hand, Roper and Zentall (1993) noted that procedures for demonstrating directed forgetting in humans may be different in an important way from the procedures that have been used with pigeons. In studies with humans, when subjects are presented with an F-cue, they 
have the opportunity to reallocate memory resources to the to-be-remembered items that have been presented previously (Johnson, 1994). In directed forgetting studies with pigeons, however, there are no alternative items to which a reallocation of memory resources can occur. In the substitution procedure, the F-cue signals a simple simultaneous discrimination, which is essentially a reference memory task that would presumably not represent a demand on the pigeons' working memory. Thus, there may be no functional benefit of forgetting the sample on F-cued trials.

In an attempt to make the directed forgetting task with pigeons more analogous to directed forgetting tasks used in human research, Roper, Kaiser, and Zentall (1995) used a procedure that allowed pigeons to reallocate memory maintenance capacity on F-cued trials. In this procedure, two F-cues were used (e.g., circle and dot shapes). These F-cues, which signaled that memory for the sample would not be tested, also served as samples in a symbolic matching task. When a circle shape was presented as the F-cue, the pigeons were reinforced for choice of a vertical lines comparison, and when a dot shape was presented as the F-cue, the pigeons were reinforced for choice of a horizontal lines comparison (see Figure 1). Thus, the F-cue not only signaled that sample memory would not be tested, but also served as a conditional stimulus to which memory could be reallocated. For symmetry, two R-cues (e.g., blue and white hues) were also used, but correct responding was independent of the identity of the $R$-cue. The R-cues merely signaled that sample memory would be tested.

This reallocation procedure controls for the nonmemorial artifacts described above, because there is opportunity for reinforcement following the F-cue and because the choice behavior on F-cue trials in training should result in a compatible response pattern for F-cued probe trial responding. Most importantly, this procedure is more analogous to procedures used to demonstrate directed forgetting in humans, because the pigeons have the opportunity to reallocate memory resources.

Using this reallocation procedure, Roper et al. (1995) found a significant decrease in performance on F-cued probe trials as opposed to R-cued trials. It appeared that when nonmemorial artifacts were controlled and pigeons were encouraged to reallocate their memory resources on F-cued trials, directed forgetting effects could be demonstrated. On the basis of these results, Roper et al. concluded that pigeons could exert active control over memory maintenance processes. It appeared that the pigeons had learned the meaning of the F-cues, and that on the Fcued trials they had shifted memory resources from the original sample to the delay stimulus (the new sample).

It is possible, however, that the experiment reported by Roper et al. (1995) suffered from a new nonmemorial artifact. Grant (personal communication, August 1995) argued that the finding reported by Roper et al. might have resulted from interference (rather than reallocation) between the F-cue and sample memory that occurred on F- cued trials but not on R-cued trials. According to this interpretation, the pigeons were not demonstrating active control over memory processing. Rather, the pigeons could have been passively processing the sample on both $\mathrm{R}$ - and F-cued trials. On F-cued trials, the pigeons had to process both the sample stimulus and the delay stimulus, whereas on R-cued trials, after the pigeons identified the delay stimulus as an R-cue, no further processing of that stimulus was necessary. Thus, R-cues ought to have interfered with memory considerably less than F-cues. This posited differential interference, produced by differential processing of R- and F-cues, may account for decreased matching accuracy on F-cued probe trials, rather than the reallocation of memory-maintaining capacity.

The purpose of the present experiment was to test for directed forgetting in pigeons while controlling for the nonmemorial artifacts described above, as well as for the possible interference effects that processing of the F-cue might cause. Pigeons were trained with a procedure similar to that used by Roper et al. (1995). However, to ensure that the pigeons processed the $\mathrm{R}$-cues as well as the F-cues, on half of the R-cued trials they were required to match the R-cue to new comparisons. If processing the F-cue delay stimulus interferes with sample memory, it was expected that when the present procedure was used, comparable interference would occur on R-cue trials. To control for the possibility that unexpected (or surprising) comparisons (presented on F-cue probe trials) might result in some disruption of matching accuracy independently of the effects of the F-cue on memory, a control group was included in the present experiment. For pigeons in the control group, analogous to the substitution control from earlier research, F-cues signaled presentation of a simple simultaneous discrimination. Thus, for the control group, F-cues would not be associated in training with memory reallocation, but as with the experimental group, they would involve similar effects of novel comparisons on F-cue probe trials.

If under these conditions directed forgetting could be induced in pigeons in the experimental group, there would be a significant difference in matching accuracy for the experimental group between remember (matching) trials and forget-probe trials. Furthermore, the magnitude of that directed forgetting effect would be significantly greater for pigeons in the experimental group than in the control group (i.e., there would be a significant group $\times$ trial type interaction).

\section{METHOD}

\section{Subjects}

Thirteen White Carneaux pigeons obtained from the Palmetto $\mathrm{Pi}$ geon Plant (Sumter, SC) were used as subjects. They had had previous experience with simple simultaneous discriminations. The pigeons were maintained at $80 \%$ of their free feeding weights on a diet of Purina Pro Grains for Pigeons, which was provided primarily during experimental sessions. The pigeons were housed in individual cages in a temperature-controlled colony room and were maintained on a 12:12-h light:dark cycle. Water and grit were provided ad lib in the home cage. 

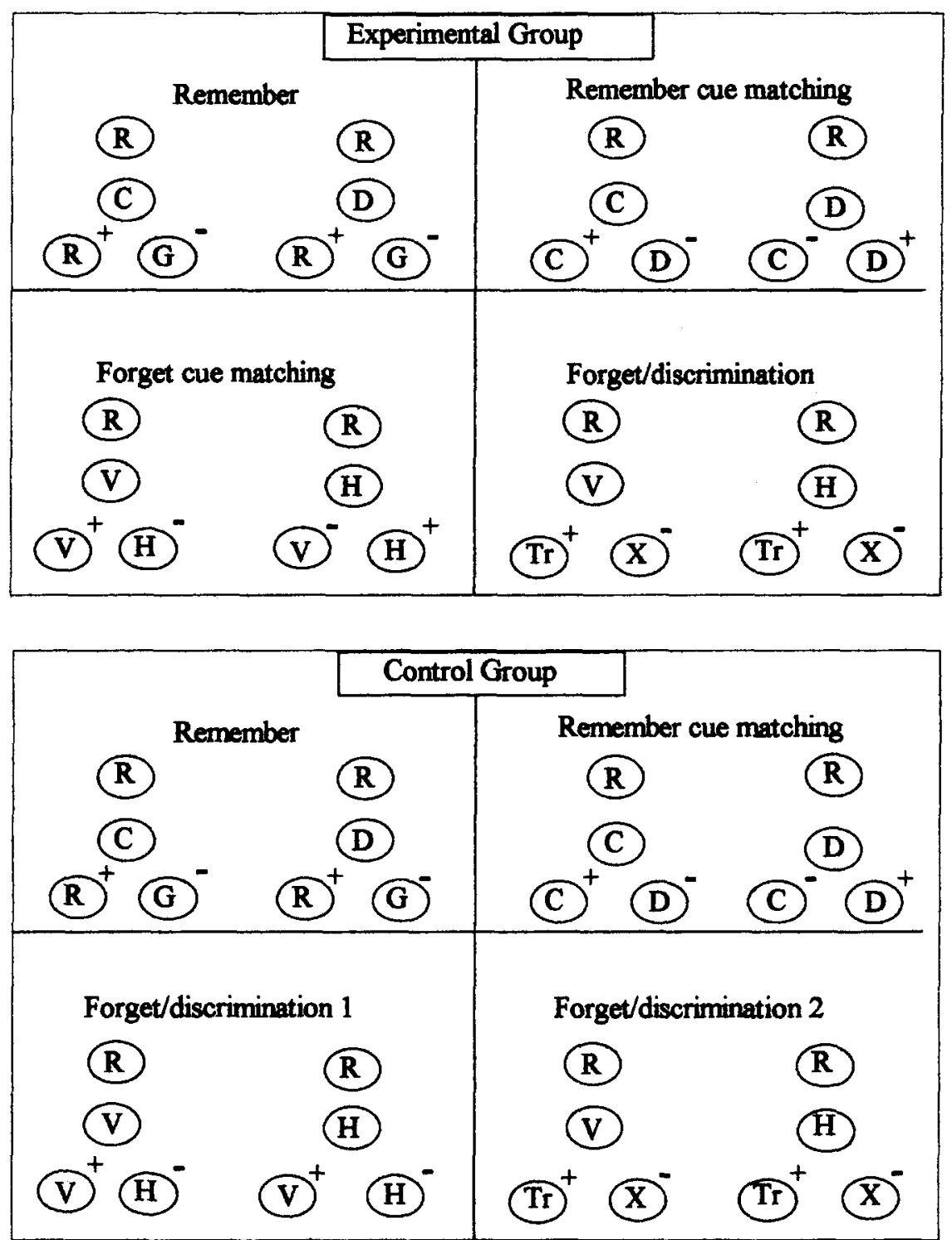

Figure 2. Representation of the procedure for the experimental and control groups. Note that only half of the trial types are presented. The other half are similar but involve green samples, and the correct comparison is green where appropriate. $R=\operatorname{red}, G=\operatorname{green}, C=\operatorname{circle}, D=\operatorname{dot}, V=$ vertical lines, $H=$ horizontal lines, $\operatorname{Tr}=$ triangle, $(+)=$ reinforcement, and $(-)=$ nonreinforcement.

\begin{abstract}
Apparatus
The experiment was conducted in a sound-attenuated BRS/LVE (Laurel, MD) operant chamber. The chamber was $31.5 \mathrm{~cm}$ high, $35.5 \mathrm{~cm}$ side to side, and $32 \mathrm{~cm}$ from the front panel to the back wall. Three square response keys $(2.5 \times 2.5 \mathrm{~cm})$, separated from each other by $1.5 \mathrm{~cm}$, were centered on the response panel with their bottom edges $20 \mathrm{~cm}$ from the wire mesh floor. Behind each key was an in-line projector (Industrial Electronics Engineering, Series 10, Van Nuys, CA, with No. 1820 G. E. lamps). Each projector could project red and green hues (Kodak Wratten filter Nos. 26 and 60, respectively), vertical and horizontal lines (three lines $2.4 \mathrm{~cm}$ long, $0.3 \mathrm{~cm}$ wide, and $0.3 \mathrm{~cm}$ apart), and a dot and circle shape (a solid area $5 \mathrm{~mm}$ in diameter, and an annulus with a $16-\mathrm{mm}$ outside diameter and a $13-\mathrm{mm}$ inside diameter, respectively). The two side projectors could also project an $\times$ (diagonal lines, each $13 \mathrm{~cm}$ long and $3 \mathrm{~mm}$ wide), and a solid equilateral triangle (apex down with $1-\mathrm{cm}$ sides). All line and shape stimuli were white and were projected on a black background Reinforcement was provided through an illuminated $5 \times 6 \mathrm{~cm}$ aper-
\end{abstract}

ture centered on the response panel. The bottom edge of the aperture was $6 \mathrm{~cm}$ from the floor. General chamber illumination was provided by a shielded houselight that was centered on the response panel, $6 \mathrm{~cm}$ above the center response key. An exhaust fan and white noise (at $72 \mathrm{~dB}$ ) masked extraneous sounds. The events in the operant chamber were controlled by a microcomputer located in an adjacent room.

\section{Procedure}

Because all pigeons had previous experience in pecking lit keys, they were immediately placed on the matching-to-sample task. Each trial began with the presentation of a red- or green-hue sample stimulus on the center key. Pecking the center key 20 times resulted immediately in the darkening of the center key and the lighting of the two side keys with red and green comparison stimuli. A single peck to the matching comparison stimulus resulted in $1.5 \mathrm{sec}$ of reinforcement, terminated the stimulus display, and initiated a 10-sec ITI (during which the houselight was lit). A single peck to the nonmatching comparison terminated the stimulus display and initiated the ITI without 


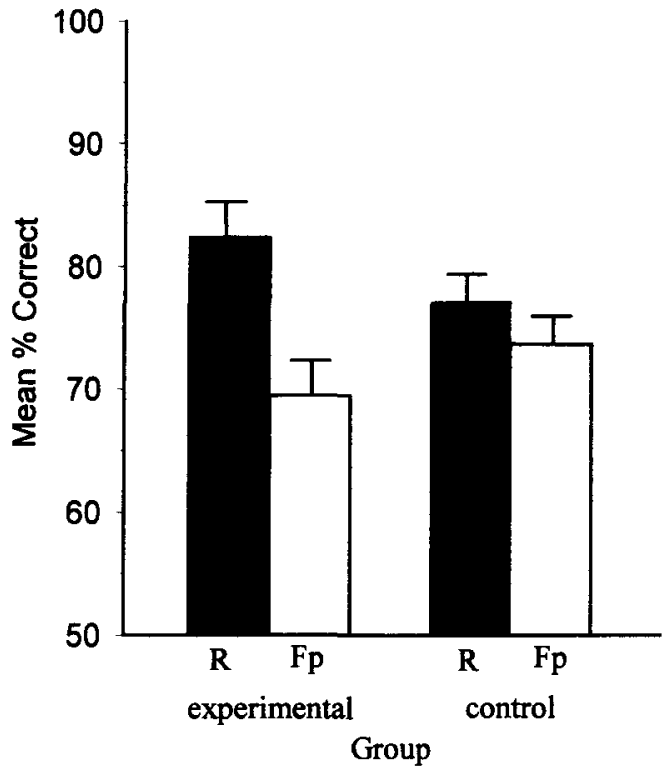

Figure 3. Mean percent correct (with standard error of the mean derived from within-subjects difference scores) on remember task trials (R) and F-cued probe trials (Fp) for the experimental and control groups.

reinforcement. The pigeons were never reinforced more than three times for consecutive choices of a given side key. The presentation of the red and green sample stimuli was randomized across trials, with the restriction that no more than three consecutive presentations of a given sample stimulus could occur, and the position of red and green comparisons was balanced so that red and green appeared on the left and right keys equally often across trials and did not occur on the same key for more than three consecutive trials. The pigeons received 96 trials per session ( 48 red and 48 green sample trials), and they were trained in this manner until they reached an individual performance criterion of 2 consecutive days with at least $90 \%$ correct comparison choices. As they reached this initial performance criterion, the pigeons were divided into two groups in order to approximately equate group membership for sessions to criterion on the initial red/green matching task.

Experimental group. After initial red/green matching training, the pigeons assigned to the experimental group $(n=6)$ were trained on the entire experimental task with 1 -sec delays between samples and comparisons, during which a delay stimulus was presented on the center key. There were four "problems," which were presented randomly within each 96-trial session ( 24 trials with each problem). A schematic of the four training problems for the experimental group appears in the top panel of Figure 2 . All trials began with the presentation of the red or green sample stimulus on the center key. Twenty responses to the center key resulted in the replacement of the sample stimulus with an $\mathrm{R}$ - or F-cue delay stimulus on the center key. On half of the R-cue trials (remember), the R-cue delay stimulus (i.e., circle or dot) was followed by the presentation of comparisons, and reinforcement was contingent on a response to the stimulus that matched the initial sample (red or green). On the other half of the R-cue trials (remember cue matching), the R-cue delay stimulus (i.e., circle or dot) was followed by the presentation of comparisons, and reinforcement was contingent on a response to the stimulus that matched the R-cue (i.e., circle or dot). On half of the F-cue trials (forget cue matching), the F-cue delay stimulus (i.e., vertical or horizontal lines) was followed by the presentation of comparisons, and reinforcement was contingent on a response to the stimulus that matched the F-cue (i.e., vertical or horizontal lines). To control for differences in comparison predictability that could occur if one of two comparison pairs could be presented following an R-cue but only one comparison pair could be presented following an F-cue, additional F-cue trials (forget/discrimination) were added. On these additional trials, the F-cue delay stimulus was followed by presentation of a simple simultaneous discrimination (triangle and $\times$ shape stimuli) in which one stimulus (e.g., triangle) was always reinforced, and the other stimulus (e.g., $x$ ) was never reinforced, regardless of the identity of the sample or delay stimulus. Rand F-cues were counterbalanced so that circle and dot as well as vertical and horizontal lines served as both $\mathrm{R}$ - and $\mathrm{F}$-cues over pigeons. When the pigeons reached a criterion of at least $90 \%$ correct choices on remember (sample memory) trials, the delay was increased to $2 \mathrm{sec}$, and when they reached this criterion with $2-\mathrm{sec}$ delay stimuli, the delay was increased to $4 \mathrm{sec}$. The pigeons began sessions with $\mathrm{F}$ cued probe trials after 20 sessions of 4-sec delay training if they had achieved at least $90 \%$ correct matching accuracy on each of the problems (sample and delay cue matching) at some point during 4-sec delay training and had maintained at least $80 \%$ correct matching on all four problems on the previous session. Pigeons that were not performing well on the remember task after 20 sessions of delay training were placed on a repeat-trial correction procedure, in which incorrect responding resulted in the maintenance of the comparisons until there was no responding for $2 \mathrm{sec}$ (penalty-time; see Strength \& Zentall, 1991). For pigeons that still had difficulty with a particular problem, that problem was presented in the absence of the others until the pigeons reached $90 \%$ correct. They were then returned to the four-problem procedure and were required to meet criterion.

Control group. On R-cued trials, the control group $(n=7)$ was treated identically to the experimental group (see Figure 2), with the exception that on F-cued trials pigeons in the control group were not required to match comparisons to the $\mathrm{F}$-cue. Rather, following each $\mathrm{F}$ cue, these pigeons were presented with one of two simple simultaneous discriminations. Thus, the only difference between the experimental group and the control group was that the experimental group was required to match comparisons (e.g., vertical and horizontal lines) to the F-cue delay stimulus (e.g., vertical or horizontal lines) on half of the F-cued trials and received a simple simultaneous discrimination following the F-cue on the other half of the F-cued trials, whereas the control group received two different simple simultaneous discriminations following the F-cue, one involving vertical and horizontal line stimuli (e.g., vertical lines were always correct, regardless of the delay cue), and one involving triangle and $\times$ shape stimuli (e.g., the triangle was always correct, regardless of the delay cue). Prior to being tested, the pigeons in the control group were required to meet the same performance criterion as that for the pigeons in the experimental group, and they were also subjected to the same correction procedures when necessary.

Test sessions. The test sessions were identical to delay cue training sessions, with the exception that four probe trials were added per session (one within each block of 24 trials). The probe trials involved presentation of the sample (red or green) followed by an F-cue, and then presentation of red and green comparison stimuli. There were eight possible probe trials ( 2 samples $\times 2$ F-cues $\times 2$ comparison locations). Thus, the pigeons received all eight of the possible probe trials over two consecutive sessions. The experimental and control groups received identical probe trials. All the pigeons were tested with probe trials for 25 sessions.

\section{RESULTS}

The 6 pigeons in the experimental group and 7 in the control group did not show a significant difference in acquisition of the delayed matching task from initial red/ green matching to the start of test [ 64.5 and 87.1 sessions, respectively; $F(1,12)<1]$. Data from probe-trial sessions pooled over the 25 test sessions appear in Figure 3. Typically, the appropriate comparison for directed forgetting is the within-subjects trial-type effect. In the present experiment, however, the relative magnitude of the withinsubjects trial-type effect for the experimental and control 
groups is critical. Thus, the probe session data were analyzed with a two-way mixed analysis of variance with group and remember-trial versus F-cued probe-trial matching accuracy as factors. This analysis revealed a significant main effect of remember- versus probe-trial performance $[F(1,11)=25.94, p<.001]$ and a significant group $\times$ remember- versus probe-trial matching accuracy interaction $[F(1,11)=8.81, p<.02]$, but no significant main effect of group. The significant interaction of group $\times$ trial type indicates that the directed forgetting effect was significantly greater in the experimental group than in the control group. Simple main effects analyses of this interaction revealed that for the experimental group, matching accuracy was significantly poorer on probe trials than on remember trials $[F(1,5)=$ $30.16, p<.01]$. The control group also showed a small difference in matching accuracy on F-cued probe trials relative to remember trials, but this difference was not significant $[F(1,6)=2.45, p>.05]$.

\section{DISCUSSION}

We conducted the present experiment to ask whether pigeons would be capable of active control over memory maintenance when they were tested with procedures that both controlled for possible procedural artifacts and were more analogous to those used in human directed forgetting studies than procedures used in previous animal studies. Specifically, this experiment was designed to address a concern that in a previous examination of the effects of reallocation of memory in a directed forgetting paradigm (Roper et al., 1995), the effects reported might have been due to differential interference produced by R- and F-cue delay stimuli. In the present experiment, differential processing of delay stimuli on $R-$ and F-cue trials was controlled, and a reliable distuption in matching accuracy on F-cued probe trials (as compared with R-cued trials) was found. Hence, in the present experiment, the obtained directed forgetting effect strongly suggested that active control over memorial processing can be demonstrated in pigeons. Thus, the present findings support the notion that when procedures are used that allow for reallocation of memory, pigeons are capable of actively controlling memorial processes in a fashion analogous to that seen in human directed forgetting research.

These findings also suggest that the decrement in performance on probe trials that was observed in the Roper et al. (1995) study was unlikely to have been produced by differential interference effects on Rand F-cued trials. In the present experiment, there was about a 13\% decrement in matching accuracy on $\mathrm{F}$-cued probe trials in comparison with R-cued trials, a decrement comparable to that found by Roper et al.

It should be noted that Grant and Soldat (1995) have reported evidence for directed forgetting in pigeons, using a successive matchingto-sample procedure that appears to have controlled for all of the procedural artifacts described here, but without using a reallocation manipulation. In successive matching-to-sample, a single comparison stimulus follows each sample, and pecks to the comparison stimulus are reinforced only if that comparison matches the original sample. Directed forgetting can be examined with this procedure by inserting delay stimuli between sample offset and comparison onset in the same way as has been discussed earlier with delayed matching-to-sample using two simultaneously presented comparisons. Grant and Soldat successfully controlled for reinforcement and response compatibility by following $F$-cues with a simple successive discrimination. This procedure provided for reinforcement on F-cued trials and also allowed for a similar response pattern following R- and F-cues (i.e., go/no go). Thus, the decrement in performance on probe trials that they reported cannot be accounted for by these factors. Although Grant and Soldat's results suggest evidence for directed forgetting in the absence of real- location procedures, Grant and Soldat may not have adequately controlled for the novelty of the unexpected comparisons that served as a test of memory on the F-cued probe trials. In the experiment reported here, only a small (nonsignificant) effect of comparison novelty was found in the control group. With the choice procedure used here, however, if the pigeons are surprised by unexpected comparisons, they still must choose between comparisons for reinforcement. On the other hand, in successive matching, the novelty of comparison presentation on F-cue probe trials may result in the disinhibition (or recovery) of responding on nonreinforced trials. To anticipate this criticism, Grant and Soldat cited Santi, Musgrave, and Bradford (1988), who found (consistent with the results of the present experiment) that in a one-tomany matching-to-sample procedure in which delay stimuli were used to designate which comparison pair would be presented, there was very little decrement in matching accuracy on probe trials when the delay stimuli miscued the comparison pairs. However, Santi et al. used a choice procedure with simultaneous presentation of comparisons, similar to that used in the present experiment. Grant and Soldat's conclusions would be more convincing if a control design had been used that was similar to Santi et al.'s procedure, but that involved a successive matching-to-sample procedure. If, under such conditions, one could show very little decrement in matching accuracy when comparison stimuli were miscued, the conclusion that unexpected comparisons could not account for the decrement in performance on probe trials in the Grant and Soldat study wuuld be more directly supported.

Regardless of whether more traditional procedures can be used to demonstrate true directed forgetting in pigeons, the reallocation procedures presented here (see also Roper et al., 1995) may provide a better analogue of human research in directed forgetting. For this reason, we would recommend that reallocation procedures be used in future research addressing issues of active control over memory maintenance by animals.

\section{REFERENCES}

BJORK, R. A. (1972). Theoretical implications of directed forgetting. In A. W. Melton \& E. Martin (Eds.), Coding processes in human memory (pp. 217-235). New York: Winston \& Wiley.

EPSTEIN, W. (1972). Mechanisms of directed forgetting. In G. H. Bower (Ed.), The psychology of learning and motivation (Vol. 6, pp. 147-191). New York: Academic Press.

GRANT, D. S. (1981). Stimulus control of information processing in pigeon short-term memory. Learning \& Motivation, 12, 19-39.

GRANT, D. S. (1982). Stimulus control of information processing in rat short-term memory. Journal of Experimental Psychology: Animal Behavior Processes, 8, 154-164.

Grant, D. S., \& Soldat, A. S. (1995). A postsample cue to forget does initiate an active forgetting process in pigeons. Journal of Experimental Psychology: Animal Behavior Processes, 3, 218-228.

Hull, C. L. (1943). Principles of behavior. New York: AppletonCentury-Crofts.

JoHnson, H. M. (1994). Processes of successful intentional forgetting. Psychological Bulletin, 116, 274-292.

Kendrick, D. F., Rilling, M., \& Stonebraker, T. B. (1981). Stimulus control of delayed matching in pigeons: Directed forgetting. Journal of the Experimental Analysis of Behavior, 36, 241-251.

MAKI, W. S., \& HEGVIK, D. K. (1980). Directed forgetting in pigeons. Animal Learning \& Behavior, 8, 567-574.

MAKI, W. S., Olson, D., \& REgo, S. (1981). Directed forgetting in pigeons: Analysis of cue functions. Animal Learning \& Behavior, 9. 189-195.

RoberTs, W. A., \& GRant, D. S. (1976). Studies of short-term memory in the pigeon using the delayed matching-to-sample procedure. In D. L. Medin, W. A. Roberts, \& R. T. Davis (Eds.), Processes of animal memory (pp. 79-112). Hillsdale, NJ: Erlbaum.

Roberts, W. A., Mazmanian, D. S., \& Kraemer, P. J. (1984). Directed forgetting in monkeys. Animal Learning \& Behavior, 12, 29-40.

RoItBl.AT, H. L. (1982). The meaning of representations in animal memory. Behavioral \& Brain Sciences, 5, 353-406. 
Roper, K. L., KaIser, D. H., \& Zentall, T. R. (1995). True directed forgetting in pigeons may occur only when alternative working memory is required on forget-cue trials. Animal Learning \& Behavior, 23, 280-285.

RoPER, K. L., \& ZENTALL, T. R. (1993). Directed forgetting in animals Psychological Bulletin, 113, 513-532.

Santi, A., Musgrave, S., \& Bradford, S. A. (1988). Utilization of cues signaling different test stimulus dimensions in delayed matching to sample by pigeons. Learning \& Motivation, 19, 87-98.

SANTI, A. \& SAVICH, J. (1985). Directed forgetting effects in pigeons: Remember cues initiate rehearsal. Animal Learning \& Behavior, 13, 365-369.

Stonebraker, T. B., \& Rilling, M. (1981). Control of delayed matching-to-sample performance using directed forgetting techniques. Animal Learning \& Behavior, 9, 196-201.
Strength, V., \& Zental., T. R. (1991). Matching and oddity learning in pigeons: Effects of penalty time for incorrect responding. $A n$ imal Learning \& Behavior, 19, 49-57.

Terrace, H. S. (1971). By-products of discrimination learning. In G. H. Bower (Ed.), The psychology of learning and motivation (Vol. 5, pp. 195-265). New York: Academic Press.

Zentall, T. R., Roper, K. L., \& Sherburne, L. M. (1995), Most directed forgetting in pigeons can be attributed to the absence of reinforcement on forget trials during training or to other procedural artifacts. Journal of the Experimental Analysis of Behavior, 63, 127-137.

(Manuscript received December 24, 1996; revision accepted for publication April 24, 1997.) 